\title{
Gauge invariance in the process $e^{+} e^{-} \rightarrow \bar{\nu}_{e} e^{-} W^{+} \rightarrow \bar{\nu}_{e} e^{-} u \bar{d}$
}

\author{
Robin G. Stuart \\ Randall Laboratory of Physics, University of Michigan, Ann Arbor, MI 48109-1120, USA
}

Received: 1 March 1997 / Revised version: 19 September 1997 / Published online: 26 February 1998

\begin{abstract}
The process $e^{+} e^{-} \rightarrow \bar{\nu}_{e} e^{-} W^{+} \rightarrow \bar{\nu}_{e} e^{-} u \bar{d}$ is considered as an example of the problems associated with maintaining gauge invariance in matrix elements involving unstable particles. It is shown how to construct a matrix element that correctly treats width effects for the intermediate unstable $W$ boson

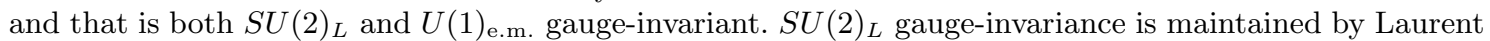

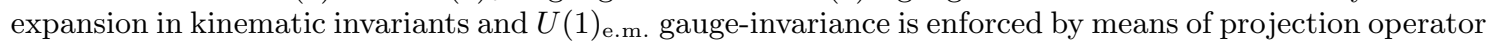
under which the exact matrix element is invariant.
\end{abstract}

\section{Introduction}

In recent years the difficulty and importance of producing exactly gauge-invariant amplitudes for processes involving unstable particles has come to be appreciated. Prior to 1991 all calculations of electroweak physics at the $Z^{0}$ resonance, and even the definition of $M_{Z}$, were gaugedependent. A variety of techniques have been applied to try to remove explicit gauge-dependence. Some of these involve the generation of gauge-invariant Green's functions, such as self-energies and vertex corrections [1-3]. Such techniques however replace dependence on, say, the gauge parameter by some arbitrary choice in the definition of the Green's function. In the case of the so-called 'pinch technique' the procedure used may be justified by the background field method $[4,5]$ for a particular choice of the gauge parameter, $\xi$. The fact that a particular choice is involved raises questions as to whether the gauge dependence has been removed or merely disguised.

A method for producing genuinely gauge-invariant amplitudes at all orders in perturbation theory is that of Laurent expansion as proposed in $[6,7]$. This method starts from the general structure of the exact physical $S$-matrix element for the process under consideration. A Laurent expansion then decomposes this expression into resonant and non-resonant pieces that must be separately gaugeinvariant provided the initial physical matrix element is. The question of whether this process always leads to results with good high-energy behaviour, as is apparently the case for the process considered here, requires further study and has been discussed in [8]. In [9] it was pointed out that the expansion was not just a mathematical trick. The leading resonant term in the expansion represents the finite propagation and subsequent decay of a physical unstable particle. As such it constitutes a distinguishable physical process and therefore must be exactly gaugeinvariant. This insight was used to give an answer to the long-standing problem of how to calculate production cross-sections for unstable particles and was applied to the process $e^{+} e^{-} \rightarrow Z^{0} Z^{0}$.

The presence of an unstable particle is indicated by a finite propagation length separating production and decay vertices. The contributions responsible for finite propagation are just those that are resonant in the invariant mass of the decay products. Thus, for example, to calculate the cross-section $e^{+} e^{-} \rightarrow Z^{0} Z^{0}$ one calculates the matrix element for the process $e^{+} e^{-} \rightarrow Z^{0} Z^{0} \rightarrow\left(f_{1} \bar{f}_{1}\right)\left(f_{2} \bar{f}_{2}\right)$ and extracts the part resonant in the invariant masses, $p_{1}^{2}$ and $p_{2}^{2}$, of the $\left(f_{1} \bar{f}_{1}\right)$ and $\left(f_{2} \bar{f}_{2}\right)$ pairs respectively. This is accomplished by means of a Laurent expansion in each of the variables $p_{1}^{2}$ and $p_{2}^{2}$. In the notation of [10] the general matrix element for the process takes the form

$$
\begin{aligned}
& \mathcal{M}\left(\ldots, p_{1}, p_{2}, \ldots\right) \\
& =\sum_{i} l_{i}\left(\ldots, p_{1}, p_{2}, \ldots\right) A_{i}\left(t, u, p_{1}^{2}, p_{2}^{2}\right)
\end{aligned}
$$

where the $l_{i}$ are 'standard covariants', with external wavefunctions attached, that form a basis for the spinor and Lorentz tensor structure of the matrix element. They therefore transform as $\mathcal{M}$ does. The $A_{i}$ are Lorentz scalar functions of the independent Lorentz invariants of the problem and contain no kinematic singularities. The resonant part is extracted by taking the leading terms $\hat{A}_{i}$ in a Laurent expansion first in $p_{1}^{2}$ and then $p_{2}^{2}$.

The cross-section formed from the resonant parts

$$
\begin{aligned}
& \widehat{\mathcal{M}}\left(\ldots, p_{1}, p_{2}, \ldots\right) \\
& =\sum_{i} l_{i}\left(\ldots, p_{1}, p_{2}, \ldots\right) \hat{A}_{i}\left(t, u, p_{1}^{2}, p_{2}^{2}\right)
\end{aligned}
$$

or in this case doubly resonant parts, of the matrix element and then summed over all possible final states constitutes the cross-section $\sigma\left(e^{+} e^{-} \rightarrow Z^{0} Z^{0}\right)$. If the standard covariants, $l_{i}$, do form a basis then the scalars $A_{i}$ will be gauge-invariant and hence so will the $\hat{A}_{i}$. 
The external particle wave functions do not feature in the expansion process hence the kinematics of the problem are unchanged and momentum conservation is preserved throughout. The final state integrations involve only stable on-shell particles and so things like complex scattering angles that have plagued certain other attempts at calculating such cross-sections do not arise. Problems near 'threshold' that have been noted elsewhere $[11,12]$ do not appear. This is as expected since unstable particles do not exhibit sharp thresholds as do stable ones. Branch points corresponding to their production lie off the real axis and such threshold problems must be calculational artifacts. Because matrix elements are always evaluated with external stable states they remain gauge-invariant throughout. As guaranteed by Fredholm theory, there will be an exact factorization between the final state decay products of the unstable particle and the rest of the matrix element. Because of this exact factorization, the cross section takes the form

$$
\begin{aligned}
\sigma(s)= & \int_{0}^{s} d p_{1}^{2} \int_{0}^{\left(\sqrt{s}-\sqrt{p_{1}^{2}}\right)^{2}} \\
& \times d p_{2}^{2} \sigma\left(s ; p_{1}^{2}, p_{2}^{2}\right) \rho\left(p_{1}^{2}\right) \rho\left(p_{2}^{2}\right),
\end{aligned}
$$

where $\sigma\left(s ; p_{1}^{2}, p_{2}^{2}\right)$ is the cross-section obtained from the resonant part of the matrix element alone and

$$
\rho\left(p^{2}\right) \approx \frac{1}{\pi} \cdot \frac{p^{2}\left(\Gamma_{Z} / M_{Z}\right)}{\left(p^{2}-M_{Z}^{2}\right)^{2}+\Gamma_{Z}^{2} M_{Z}^{2}} \theta\left(p_{0}\right) \theta\left(p^{2}\right)
$$

is Breit-Wigner-like convolution function.

In [13] the process $e^{+} e^{-} \rightarrow \bar{\nu}_{e} e^{-} W^{+} \rightarrow \bar{\nu}_{e} e^{-} u \bar{d}$ is treated as an example of how to include width-effects in matrix elements involving unstable particles. Much is made of electromagnetic $U(1)_{\text {e.m. }}$ gauge invariance and the possibility that the large mass ratio $s / m_{e}^{2}$ present in this process might amplify uncancelled gauge-dependence to a disastrous level $[14,15]$. In particular it is shown that gauge invariance guarantees that the cross-section behaves as $\sim q^{-2}$ as $q^{2} \rightarrow 0$ and not $\sim q^{-4}$ as it might otherwise do. However the question of how to simultaneously maintain $S U(2)_{L}$ and $U(1)_{\text {e.m. }}$ gauge invariance in calculations that take account of $W$ boson width effects is not considered. The approach advocated is to include a subset of higher-order corrections to restore $U(1)_{\text {e.m. }}$ gauge invariance as was done in $[16,17]$. This procedure is however inconvenient, difficult to apply consistently and adds considerably to the calculational labour involved. As noted in [13] the reparation scheme is arbitrary.

More recently the above method has been extended to encompass all diagrams containing a single fermion loop [18]. This represents a complete self-consistent $\mathcal{O}\left(N_{f} \alpha\right)$ calculation where $N_{f}$ is the number of fermions. However it is also known that this approach cannot maintain $S U(2)_{L}$ gauge-invariance when 1-loop bosonic corrections are included. This can be especially serious at high energies where strong unitarity cancellations occur.

It may be commented that the use of next order diagrams to fix problems at a given order for processes involving unstable particles has been noted elsewhere [19].
In that case troublesome imaginary parts of counterterms were observed to be canceled by next order diagrams.

\section{Gauge invariance for massless particles}

When massless gauge particles are present special problems arise. Suppose we were to write a Green's function $G^{\mu}$ with a photon leg in terms of some basis of standard covariants, $l_{i}^{\mu}$, and Lorentz scalars, $A_{i}$, in a form analogous to (1) and (2),

$$
\begin{aligned}
& G^{\mu}\left(p_{1}, p_{2}, \ldots\right) \\
& \quad=\sum_{i} l_{i}^{\mu}\left(p_{1}, p_{2}, \ldots\right) A_{i}\left(p_{1}^{2}, p_{2}^{2}, \ldots\right) .
\end{aligned}
$$

The $l_{i}^{\mu}$, of course, must transform in the same way that $G^{\mu}$ does. There is a certain amount of freedom as to what to include in the standard covariants, $l_{i}^{\mu}$, and what in the Lorentz scalars, $A_{i}$, but, provided one incorporates all analytic dependence on the expansion variable in the $A_{i}$ 's and does not introduce artificial singularities, the final results for physical observables cannot be affected.

The gauge condition on Green's functions $q_{\mu} G^{\mu}=0$ means that the $A_{i}$ are not linearly independent since they satisfy the condition $\sum_{i}\left(q \cdot l_{i}\right) A_{i}=0$ where $q_{\mu}$ is the momentum of the photon leg.

Gauge-invariant Green's functions can be obtained using the methods of Bardeen and Tung [10]. Suppose that we have some exact Green's function expressed in terms of standard covariants and Lorentz scalars as in (4). Since $q_{\mu} G^{\mu}=0$ the Green's function is invariant under of the action of the operator

$$
I_{\nu}^{\mu}=g_{\nu}^{\mu}-\frac{p^{\mu} q_{\nu}}{p \cdot q}
$$

where $p$ is some conveniently chosen momentum. The spinor-Lorentz tensor structure of the Green's function is spanned by the set $\left\{I_{\nu}^{\mu} l_{i}^{\nu}\right\}$. That is

$$
\begin{aligned}
& G^{\mu}\left(p_{1}, p_{2}, \ldots\right) \\
& \quad=\sum_{i}\left(I_{\nu}^{\mu} l_{i}^{\nu}\left(p_{1}, p_{2}, \ldots\right)\right) A_{i}\left(p_{1}^{2}, p_{2}^{2}, \ldots\right) .
\end{aligned}
$$

For an approximate Green's function that does not satisfy the $U(1)_{\text {e.m. }}$ gauge-condition, the operator $I_{\nu}^{\mu}$ serves as a projection operator onto $\left\{I_{\nu}^{\mu} l_{i}^{\nu}\right\}$. From the $\left\{I_{\nu}^{\mu} l_{i}^{\nu}\right\}$ it is always possible to construct a new basis that is free of kinematic singularities that might have occurred when, for example, $(p \cdot q)=0$.

In general consider a Green's function $G^{\mu}$ calculated using some incomplete expansion up to some order. It will consist of two parts

$$
G^{\mu}=G_{0}^{\mu}+G_{1}^{\mu}
$$

where $G_{0}^{\mu}$ is a consistent gauge-invariant contribution correct to the given order of the calculation. $G_{1}^{\mu}$ is a spurious higher-order gauge-dependent correction. Because $G_{0}^{\mu}$ satisfies the gauge condition, $q_{\mu} G_{0}^{\mu}=0$, it is invariant under 

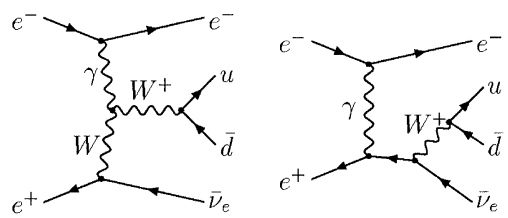

Fig. 1. Tree-level diagrams contributing to the resonant part of the process $e^{+} e^{-} \rightarrow \bar{\nu}_{e} e^{-} W^{+} \rightarrow \bar{\nu}_{e} e^{-} u \bar{d}$

the action of the operator $I_{\mu}^{\nu}$. Thus at a given order we may make the replacement

$$
G^{\mu} \rightarrow I_{\nu}^{\mu} G^{\nu}=G_{0}^{\mu}+I_{\nu}^{\mu} G_{1}^{\nu}
$$

Only $G_{1}^{\mu}$ is affected by the projection operator but since it is of higher order this is of no concern. The important point is that it now satisfies the gauge condition $q_{\mu}\left(I_{\nu}^{\mu} G_{1}^{\nu}\right)=0$ and is prevented from causing too strong a numerical blow up as $q^{2} \rightarrow 0$.

\section{The process $\mathrm{e}^{+} \mathbf{e}^{-} \rightarrow \bar{\nu}_{\mathrm{e}} \mathbf{e}^{-} \mathbf{W}^{+} \rightarrow \bar{\nu}_{\mathbf{e}} \mathbf{e}^{-} \mathbf{u} \overline{\mathbf{d}}$}

Consider the process $e^{+} e^{-} \rightarrow \bar{\nu}_{e} e^{-} W^{+} \rightarrow \bar{\nu}_{e} e^{-} u \bar{d}$. The tree level diagrams contributing to this process are shown in Fig. 1. In the kinematic regions where the process is allowed (but $W^{+} W^{-}$production is forbidden) it is expected to be dominant because of the presence of a resonant $W$ propagator. We will mainly concern ourselves with the region with $q^{2}$ small but the methods used apply in any kinematic region and will yield results that are exactly $S U(2)_{L}$ and $U(1)_{\text {e.m. }}$ gauge-invariant order by order.

Let $q$ be the 4 -momentum of the virtual photon and $p_{+}$ be that of the $W^{+}$. The momenta of the incoming electron and positron are $p_{1}$ and and $k_{1}$ respectively and $p_{2}$ and $k_{2}$ are those of the outgoing electron and $\bar{\nu}_{e}$. Hence

$$
\begin{aligned}
& p_{+}=p_{u}+p_{d}, \quad p_{-}=k_{1}-k_{2}, \\
& q=p_{1}-p_{2} .
\end{aligned}
$$

$M_{W}$ is the $W$ mass, $e$ and $g$ are the electromagnetic and $S U(2)_{L}$ weak coupling constants respectively. $Q_{i}$ is the electric charge of particle $i$. As usual $\gamma_{L}=\frac{1}{2}\left(1-\gamma_{5}\right)$ is the left-handed helicity operator.

Using the same notation as in the introduction the exact matrix element takes the form

$$
\mathcal{M}=\sum_{i} l_{i}\left(q, p_{+}, \ldots\right) A_{i}\left(q^{2}, p_{+}^{2}, \ldots\right)
$$

This matrix element may be divided into a part that has a simple pole at $q^{2}=0$ and a part that has no pole

$$
\begin{aligned}
\mathcal{M}= & \sum_{i} l_{i}\left(q, p_{+}, \ldots\right) \frac{R_{i}\left(0, p_{+}^{2}, \ldots\right)}{q^{2}} \\
& +\sum_{i} l_{i}\left(q, p_{+}, \ldots\right) \frac{R_{i}\left(q^{2}, p_{+}^{2}, \ldots\right)-R_{i}\left(0, p_{+}^{2}, \ldots\right)}{q^{2}} .
\end{aligned}
$$

These terms must be separately $S U(2)_{L}$ gauge-invariant because their differing pole structures. The first term receives contributions from diagrams, such as those of Fig. 1, in which a photon is connected to the external electron current. The second term receives contributions from these as well many other topologies. Examples are the diagrams of Fig. 1 in which the photon is replaced by a $Z^{0}$ boson.

We will be interested in the small $q^{2}$ region and will thus concentrate on the first term in (10) and write

$$
\mathcal{M}=\sum_{i} J_{\mu}^{\gamma}\left(l_{i}^{\prime}\right)^{\mu}\left(q, p_{+}, \ldots\right) R_{i}^{\prime}\left(0, p_{+}^{2}, \ldots\right)
$$

where

$$
J_{\mu}^{\gamma}=\frac{V_{\gamma}(0)}{\left[1-\Pi_{\gamma \gamma}^{\prime}(0)\right]^{\frac{1}{2}}} \cdot \frac{\bar{u}\left(p_{2}\right) \gamma_{\mu} u\left(p_{1}\right)}{q^{2}} .
$$

The $\left(l_{i}^{\prime}\right)^{\mu}\left(q, p_{+}, \ldots\right)$ are standard covariants with attached external wavefunctions and $V_{\gamma}\left(q^{2}\right)$ is the $\gamma e^{+} e^{-}$vertex form-factor. $\Pi_{\gamma \gamma}^{\prime}\left(q^{2}\right)$ is the derivative of the photon selfenergy with respect to $q^{2}$. This expression ignores $Z-\gamma$ mixing that has the effect of modifying the overall multiplicative factor and may be included using the methods of [7]. The factorization of the matrix element in this way is guaranteed by Fredholm theory. Moreover the resulting factors must be separately gauge-invariant [7]. This is important for ensuring that the subsequent insertion of the projection operator, $I_{\nu}^{\mu}$, into the photon leg does not generate gauge-dependent terms. Since $\mathcal{M}$ is $U(1)_{\text {e.m. gauge }}$ invariant it is possible to find a set $\left\{\left(l_{i}^{\prime}\right)^{\mu}\left(q, p_{+}, \ldots\right)\right\}$ that is free of kinematic singularities and that satisfy $q_{\mu}\left(l_{i}^{\prime}\right)^{\mu}\left(q, p_{+}, \ldots\right)$ $=0[10]$.

From the part of the matrix element (11) we wish to extract the dominant resonant part of the matrix element that describes the production of a physical $W^{+}$and its subsequent decay into the $\bar{u} d$ final state. As with the process $e^{+} e^{-} \rightarrow Z^{0} Z^{0}$ this is the leading term in a Laurent expansion of $R_{i}\left(0, p_{+}^{2}, \ldots\right)$ in $p_{+}^{2}$, the invariant mass of the $W^{+}$decay products. The result takes the form

$$
\mathcal{M}=\sum_{i} J_{\mu}^{\gamma} J_{\alpha}^{W}\left(l_{i}^{\prime \prime}\right)^{\mu \alpha}\left(q, p_{+}, \ldots\right) R_{i}^{\prime \prime}\left(0, s_{W}, \ldots\right)
$$

where

$$
J_{\alpha}^{W}=\frac{V_{W}\left(s_{W}\right)}{\left[1-\Pi_{W W}^{\prime}\left(s_{W}\right)\right]^{\frac{1}{2}}} \cdot \frac{\bar{u}\left(p_{u}\right) \gamma_{\alpha} \gamma_{L} v\left(p_{d}\right)}{p_{+}^{2}-s_{W}}
$$

Here $\Pi_{W W}^{\prime}\left(q^{2}\right)$ is the derivative of the $W$ self energy with respect to $q^{2}$ and $s_{W}$ the solution of $p_{+}^{2}-M_{W}^{2}-$ $\Pi_{W W}\left(p_{+}^{2}\right)=0 . V_{W}\left(p_{+}^{2}\right)$ is the $W^{+} u \bar{d}$ vertex form factor. The result (13) is exactly $S U(2)_{L}$ and $U(1)_{\text {e.m. gauge- }}$ invariant.

Since the $\left(l_{i}^{\prime \prime}\right)^{\mu \alpha}\left(q, p_{+}, \ldots\right)$ are $U(1)_{\text {e.m. gauge-invariant }}$ they are invariant under the action of projection operators of the form given in (5).

In finite order calculations, because of the non-perturbative nature of the $W^{+}$resonance, $U(1)_{\text {e.m. gauge-inva- }}$ riance may not be satisfied. A gauge-invariant result may be obtained by inserting a projection operator, $I_{\nu}^{\mu}$, as defined in (5). This has the effect of discarding spurious 
gauge-dependent terms that must ultimately cancel in the final result thus avoiding the need to calculate higherorder diagrams as was done in $[13,16]$.

With these general considerations in mind we can now calculate the cross-section for the process $\sigma\left(e^{+} e^{-} \rightarrow \bar{\nu}_{e} e^{-}\right.$ $\left.W^{+} \rightarrow \bar{\nu}_{e} e^{-} u \bar{d}\right)$ in leading order and with the $W$ width effects correctly taken into account. In words this is the production cross-section for $e^{-} \bar{\nu}_{e}$ and a physical $W^{+}$that subsequently decays into a $u \bar{d}$ pair.

As discussed above the contribution relevant as $q^{2} \rightarrow 0$ takes the form

$$
\mathcal{M}=\mathcal{M}^{\mu \alpha} J_{\mu}^{\gamma} J_{\alpha}^{W}
$$

in which

$$
\mathcal{M}^{\mu \alpha}=\sum_{i=1}^{2} \mathcal{M}_{i}^{\mu \alpha} .
$$

The $\mathcal{M}_{i}^{\mu \alpha}$ correspond to the two diagrams of Fig. 1,

$$
\begin{aligned}
\mathcal{M}_{1}^{\mu \alpha}= & i e \frac{g}{\sqrt{2}} Q_{W} \frac{1}{p_{-}^{2}-M_{W}^{2}} V^{\alpha \beta \mu}\left(p_{+},-p_{-},-q\right) \\
& \times \bar{v}\left(k_{1}\right) \gamma_{\beta} \gamma_{L} v\left(k_{2}\right), \\
\mathcal{M}_{2}^{\mu \alpha}= & i e \frac{g}{\sqrt{2}} Q_{e} \bar{v}\left(k_{1}\right) \gamma^{\mu} \frac{\not k_{1}+\not 1}{\left(k_{1}+q\right)^{2}} \gamma^{\alpha} \gamma_{L} v\left(k_{2}\right) .
\end{aligned}
$$

and

$$
\begin{aligned}
V^{\mu_{1} \mu_{2} \mu_{3}}\left(p_{1}, p_{2}, p_{3}\right)= & \left(p_{1}-p_{2}\right)^{\mu_{3}} g^{\mu_{1} \mu_{2}}+\left(p_{2}-p_{3}\right)^{\mu_{1}} g^{\mu_{2} \mu_{3}} \\
& +\left(p_{3}-p_{1}\right)^{\mu_{2}} g^{\mu_{3} \mu_{1}}
\end{aligned}
$$

Note that at this order the vertices do not depend on $q^{2}$ or $p_{+}^{2}$. Thus the second term in (10) is absent. As noted earlier there is some freedom of choice as to what to assign to Lorentz covariants and what to Lorentz scalars. At this order the issue barely arises and we may simply choose $R_{i}=1$ and include everything else in the $l_{i}$ 's.

The projection operator $I_{\nu}^{\mu}$ of (5) may be inserted into (15) in order to guarantee $U(1)_{\text {e.m. gauge invariance of the }}$ Green's function, $\mathcal{M}^{\mu \alpha}$. The matrix element then becomes

$$
\mathcal{M}=\mathcal{M}^{\mu \alpha} I_{\mu}^{\lambda} J_{\lambda}^{\gamma} J_{\alpha}^{W}
$$

The matrix element squared and averaged over spins then takes the form

$$
\begin{aligned}
\left\langle|\mathcal{M}|^{2}\right\rangle & =\frac{1}{4} \sum_{e^{+} e^{-} \text {spins }} \mathcal{M}^{\mu \alpha} I_{\mu}^{\lambda} J_{\lambda}^{\gamma} \\
& \times\left(g_{\alpha \beta}-\frac{\left(p_{+}\right)_{\alpha}\left(p_{+}\right)_{\beta}}{p_{+}^{2}}\right) \rho\left(p_{+}^{2}\right) \overline{\mathcal{M}^{\nu \beta} I_{\nu}^{\rho} J_{\rho}^{\gamma}}
\end{aligned}
$$

with

$$
\begin{aligned}
& \left(g_{\alpha \beta}-\frac{\left(p_{+}\right)_{\alpha}\left(p_{+}\right)_{\beta}}{p_{+}^{2}}\right) \rho\left(p_{+}^{2}\right) \\
& =\sum_{u \bar{d} \text { spins }} \int \frac{d^{3} p_{u}}{(2 \pi)^{3} 2 p_{u}^{0}} \frac{d^{3} p_{d}}{(2 \pi)^{3} 2 p_{d}^{0}} J_{\alpha}^{W} \overline{J_{\beta}^{W}}(2 \pi)^{4} \\
& \quad \times \delta^{4}\left(p_{u}+p_{d}-p_{+}\right) \theta\left(p_{+}^{0}\right) \theta\left(p_{+}^{2}\right) \\
& =\frac{g^{2}}{48 \pi^{2}} \cdot \frac{p_{+}^{2}}{\left|p_{+}^{2}-s_{W}\right|^{2}}\left(g_{\alpha \beta}-\frac{\left(p_{+}\right)_{\alpha}\left(p_{+}\right)_{\beta}}{p_{+}^{2}}\right) \theta\left(p_{+}^{0}\right) \theta\left(p_{+}^{2}\right)
\end{aligned}
$$

at leading order. The entire effect of the Laurent expansion at this order is encapsulated in the function $\rho\left(p_{+}^{2}\right)$. At higher orders the Laurent expansion will require that vertex form factors be evaluated with complex arguments.

In forming the total production cross-section for a physical $W^{+}$'s that subsequently decays into $u \bar{d}$ we must sum over the $u \bar{d}$ final states. Hence

$$
\begin{aligned}
& \sigma\left(e^{+} e^{-} \rightarrow \bar{\nu}_{e} e^{-} W^{+} \rightarrow \bar{\nu}_{e} e^{-} u \bar{d}\right) \\
& =N_{c} \int d p^{2} \sigma\left(p^{2}\right) \rho\left(p^{2}\right) .
\end{aligned}
$$

The integral is over the full range allowed by the cut on the scattering angle, $\theta . N_{c}$ is the number of QCD colours and

$$
\begin{aligned}
\sigma\left(p^{2}\right)=\frac{1}{2 s} & \int \frac{d^{3} p_{2}}{(2 \pi)^{3} 2 p_{2}^{0}} \frac{d^{3} k_{2}}{(2 \pi)^{3} 2 k_{2}^{0}} \frac{d^{3} p_{+}}{(2 \pi)^{3} 2 p_{+}^{0}}\left\langle\left|\mathcal{M}_{0}\right|^{2}\right\rangle \\
& \times(2 \pi)^{4} \delta^{4}\left(p_{1}+k_{1}-p_{2}-k_{2}-p_{+}\right)
\end{aligned}
$$

in which

$$
\begin{aligned}
\left\langle\left|\mathcal{M}_{0}\right|^{2}\right\rangle & =\frac{1}{4} \sum_{e^{+} e^{-} \text {spins }} \mathcal{M}^{\mu \alpha} I_{\mu}^{\lambda} J_{\lambda}^{\gamma} \\
& \times\left(g_{\alpha \beta}-\frac{\left(p_{+}\right)_{\alpha}\left(p_{+}\right)_{\beta}}{p_{+}^{2}}\right) \rho\left(p_{+}^{2}\right) \overline{\mathcal{M}^{\nu \beta} I_{\nu}^{\rho} J_{\rho}^{\gamma}}
\end{aligned}
$$

and the integral is evaluated under the constraint that $p_{+}^{2}=p^{2}$. Because $\mathcal{M}^{\mu \alpha} I_{\mu}^{\lambda}$ is $U(1)_{\text {e.m. gauge-invariant, }}$ $\left\langle\left|\mathcal{M}_{0}\right|^{2}\right\rangle \sim q^{-2}$ as $q^{2} \rightarrow 0$.

Acknowledgements. The author wishes to thank D. Williams for many enlightening discussions and the authors of [13] for permitting use of their figures of Feynman diagrams.

\section{References}

1. D. C. Kennedy and B. W. Lynn, Nucl. Phys. B 322 (1989) 1

2. D. C. Kennedy, B. W. Lynn, C. J.-C. Im and R. G. Stuart, Nucl. Phys. B 321 (1989) 83

3. G. Degrassi and A. Sirlin, Phys. Rev. D 46 (1992) 3104

4. A. Denner, G. Weiglein and S. Dittmaier, Phys. Lett. B 333 (1994) 420; Nucl. Phys. B 440 (1995) 95

5. A. Denner, G. Weiglein and S. Dittmaier, in Perspectives for electroweak interactions in $e^{+} e^{-}$collisions, Ringberg Castle, Germany, 5-8 February, 1995 ed. B. A. Kniehl (World Scientific, Singapore, 1995) p. 281

6. R. G. Stuart, Phys. Lett. B 262 (1991) 113

7. R. G. Stuart, Phys. Rev. Lett. 70 (1993) 3193

8. A. Denner, S. Dittmaier and T. Hahn, Phys. Rev. D 56 (1997) 117

9. R. G. Stuart, Nucl. Phys. B 498 (1997) 28

10. W. A. Bardeen and W.-K. Tung, Phys. Rev. 173 (1968) 1423

11. A. Aeppli, F. Cuypers and G. J. van Oldenborgh, Phys. Lett. B 314 (1993) 413;

12. A. Aeppli, G. J. van Oldenborgh and D. Wyler, Nucl. Phys. B 428 (1994) 126 
13. E. N. Argyles et al., Phys. Lett. B 358 (1995) 339

14. F. A. Berends and G. B. West, Phys. Rev. D 1 (1970) 367

15. Y. Kurihara, D. Perret-Gallix, and Y. Shimizu, Phys. Lett. B 349 (1995) 367

16. U. Baur and D. Zeppenfeld, Phys. Rev. Lett. 75 (1995) 1002
17. C. G. Papadopoulous, Phys. Lett. B 352 (1995) 144

18. W. Beenakker et al., hep-ph/9612260

19. R. G. Stuart, in $Z^{0}$ Physics, Proceedings of the XXVth Rencontre de Moriond, Editions Frontières, Gif-surYvette, ed. J. Tran Thanh Vân (1990) p. 41 